\title{
Rates and Predictors of Relapse in Medication Overuse Headache: A Clinic-Based Study from China
}

\author{
Huanxian Liu Meichen Zhang $^{\mathrm{b}}$ Xiaoyan Chen ${ }^{\mathrm{a}}$ Shuping Sun ${ }^{\mathrm{a}}$ \\ Zhao Dong ${ }^{a}$ Shengyuan $\mathrm{Yu}^{\mathrm{a}}$ \\ ${ }^{a}$ Department of Neurology, The First Medical Centre, Chinese PLA General Hospital, Beijing, China; \\ ${ }^{b}$ Department of Neurology, Beijing Jingmei Group General Hospital, Beijing, China
}

\section{Keywords}

Medication overuse headache $\cdot$ Relapse $\cdot$ Risk factor

\begin{abstract}
Objective: This study aims to further assess the rates and risk factors for relapse in medication overuse headache $(\mathrm{MOH})$ patients in China. Methods: The patients were admitted to neurology outpatient clinics at Chinese PLA General Hospital (primarily for headache) and diagnosed with $\mathrm{MOH}$. They responded well to 2 months of preventive treatment and completed a 1-year consultation. General information was collected, including demographic characteristics (age, height, weight, and education level), the clinical features of the headache and the use of pain relievers (type, frequency and duration). Differences in each factor between the group with relapse and the group without relapse were analyzed by the chi-square test or Wilcoxon test. Variables with a $p<0.05$ were included as independent variables in nonconditional logistic regression analysis. Results: In total, 129 patients were recruited for this retrospective study. The relapse rate of the $\mathrm{MOH}$ patients at the 1-year follow-up was $24.8 \%$. The relapse rate was significantly higher in the patients with migraine $(29 / 97,29.9 \%)$ than in the patients with tension-type headache $(3 / 32,9.4 \%)$ and in the patients with low education levels (middle school or lower; $24 / 77,31.6 \%$ ) than in
\end{abstract}

those with high education levels (high school or higher; $8 / 52,15.4 \%)$. Nonconditional logistic regression analysis showed that the type of primary headache was an independent risk factor for $\mathrm{MOH}$ relapse (OR 3.719, $p=0.044)$. Conclusions: The relapse rate of $\mathrm{MOH}$ patients at the 1-year follow-up was $24.8 \%$ in China. The type of primary headache is an independent risk factor for $\mathrm{MOH}$ relapse, and patients with migraine as the primary headache are more prone to relapse.

(c) 2019 S. Karger AG, Basel

\section{Introduction}

Medication overuse headache (MOH) occurs in patients with preexisting primary headache due to frequent and long-term use of acute or symptomatic headache medication, which leads to aggravated headache or the emergence of new headache types [1]. According to the International Classification of Headache Disorders 3rd edition (ICHD-3), the diagnostic criteria for $\mathrm{MOH}$ include the following: (1) headache occurring on $\geq 15$ days/month in a patient with a preexisting primary headache disorder; (2) regular overuse for $>3$ months of one or more drugs that can be taken for acute and/or symptomatic treatment of headache; and (3) headache not better accounted for by an-

\section{KARGER}

๑) 2019 S. Karger AG, Basel

karger@karger.com

www.karger.com/ene
Prof. Zhao Dong

Department of Neurology, The First Medical Centre

Chinese PLA General Hospital

Fuxing Road 28, Beijing, Haidian 100853 (China)

E-Mail dong_zhaozhao@126.com 


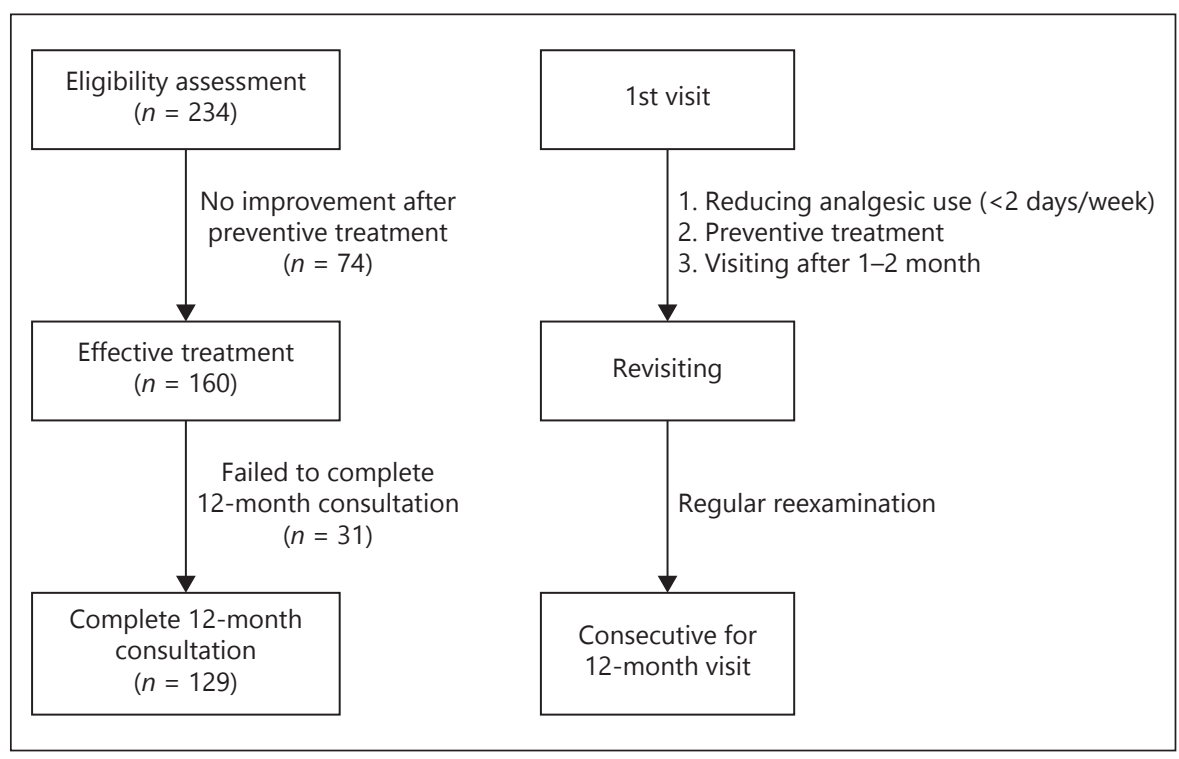

Fig. 1. Flow chart.

other ICHD-3 diagnosis [1]. The majority of studies indicate an overall prevalence of $0.5-2.6 \%$, accounting for 11$70 \%$ of patients with chronic daily headache $(\mathrm{CDH})$ [2], with epidemiology data from China showing that the $\mathrm{MOH}$ prevalence is $0.6 \%$ and that $\mathrm{MOH}$ accounts for $60 \%$ of patients with $\mathrm{CDH}$ [3]. Because $\mathrm{MOH}$ patients have headaches for $>15$ days each month and under many circumstances have daily headaches, $\mathrm{MOH}$ is a threat to public health and a disabling condition that causes a serious financial burden on society and families [4], ranking 20th in the Global Burden of Disease in 2015 [5]. MOH is often secondary to primary headaches, including migraine, tensiontype headache (TTH), and posttraumatic headache [2], and stopping or reducing the use of pain relievers to fewer than 2 days/week is an effective way to treat $\mathrm{MOH}$ [2]. However, the 1-year relapse rate for $\mathrm{MOH}$ patients undergoing drug withdrawal treatment is approximately $30 \%$ [6]. The risk factors for $\mathrm{MOH}$ relapse mainly include the type of primary headache, the type of pain reliever, combined psychological disorders, socioeconomic factors (marital status, unemployment, smoking, and drinking), and the clinical features of the headache (severity and disease course) [7]. Moreover, a clinical study by Yan et al. [8] of the risk factors reported in Chinese $\mathrm{MOH}$ patients showed that the headache frequency prior to drug withdrawal, the duration of the primary headache, and the headache frequency after drug withdrawal were independent risk factors for $\mathrm{MOH}$ relapse. However, in that study, the primary headache type was not identified as an independent risk factor for $\mathrm{MOH}$ relapse within an average of 9.5 months of follow-up and not exceeding 1 year, likely because of the limited sample size. Therefore, the present study aimed to provide comprehensive data on the 1-year relapse rate and risk factors for relapse in $\mathrm{MOH}$ patients in China.

\section{Methods}

\section{Subjects}

Patients who were admitted to the neurology outpatient clinics of Chinese PLA General Hospital and diagnosed with $\mathrm{MOH}$ for the first time between September 2015 and September 2017 were recruited in the current study. In our headache center, all $\mathrm{MOH}$ patients routinely undergo standardized evaluation and treatment, including an anxiety and depression scale assessment, basic education including an explanation of the disease etiology, a recommendation that each patient maintain a diary of their headaches, and instructions on how to correctly record information in this diary. Patients were prescribed individualized preventive drugs and instructed to limit the use of pain relievers to fewer than 2 days each week. The patients were required to return to the outpatient clinic to assess the treatment effect once every 1-2 months. The patients continued to record information in their headache diary (Fig. 1).

The inclusion criteria were as follows: (1) met the $\mathrm{MOH}$ diagnostic criteria according to the ICHD-3; (2) did not receive preventive treatment for $\mathrm{MOH}$; (3) treatment for $\mathrm{MOH}$ was effective, including the elimination or at least a $50 \%$ reduction in the frequency of headache each month after 2 months of regular preventive treatment and reduced pain reliever usage, with pain relievers being used $<10$ days/30 days; (4) visited our outpatient clinic for 1-2 months after the first visit; and (5) anxiety and depression scale testing results.

The exclusion criteria were as follows: (1) a combined history of anxiety and depression (i.e., a total score of $>6$ points on the Hamilton Anxiety scale and a total score of $>8$ points on the Hamilton Depression scale); (2) other secondary headaches; (3) comorbid diseases requiring long-term use of a pain reliever; (4) failure to continue formal $\mathrm{MOH}$ treatment for at least 2 months; or (5) 
failure to complete 12 months of visitations to our outpatient clinic or incomplete medication records. This study was approved by the Ethics Committee of Chinese PLA General Hospital. Effective $\mathrm{MOH}$ treatment was defined as complete elimination of headache following 2 months of treatment or a reduction of $50 \%$ in the number of days with headache in each month with a frequency of pain reliever use $<10$ days/30 days.

\section{Research Design}

At the first visit, all of the patients provided basic information via a unified survey, including (1) demographic characteristics (name, sex, age, height, weight, and education level); (2) the clinical features of the headache (frequency and severity); (3) the type, frequency, and duration of pain reliever use; (4) comorbid diseases, such as anxiety and depression based on the Hamilton Anxiety and Hamilton Depression scales; and (5) the patient's history of preventive treatment for their headaches. Headache severity was assessed based on the visual analog scale.

Revisiting patients provided information including (1) the frequency and severity of the primary headache, (2) the category and frequency of use of the pain reliever, and (3) the type of preventive treatment and whether medications were taken regularly. Relapse was defined according to the $\mathrm{MOH}$ diagnostic criteria in the ICHD-3. All MOH patients were divided into a relapse group and a nonrelapse group.

\section{Statistical Analysis}

All continuous measurements are presented as the mean \pm SD. Categorical variables are presented as rates. The chi-square test was performed for count data, and the Wilcoxon test was performed for nonnormally distributed continuous variables. Variables with a $p<0.05$ were included in the nonconditional logistic regression analysis. The data were analyzed using SPSS for Windows, version 21.0 (IBM Company, Chicago, IL, USA), and $p<$ 0.05 was considered indicative of statistical significance.

\section{Results}

\section{Demographic and Clinical Characteristics}

A total of 234 patients were diagnosed with $\mathrm{MOH}$ according to the ICHD-3 criteria [1]. About 1 or 2 months after individualized treatment, the patients again visited our outpatient clinic for a first consultation visit. Treatment efficacy was assessed on the basis of the headache diary or medical records, and the treatment was found to be effective in 160 patients. According to their medical records, 31 patients failed to complete 12 months of consultation (19.4\%; Fig. 1).

In total, $129 \mathrm{MOH}$ patients received effective treatment and completed the 12-month consultation period. Of these, 29 were male $(22.5 \%)$ and 100 were female $(77.5 \%)$, with an average age of $47.9 \pm 9.9(13-76)$ years. Among all $\mathrm{MOH}$ patients, 97 had migraine (97/129, 75.2\%), and 32 had TTH $(32 / 129,24.8 \%)$. The average headache duration was $16.3 \pm 10.4$ years ( $1-40$ years). The average duration
Table 1. The relationship between 1-year relapse of $\mathrm{MOH}$ in patients after treatment by primary headache type $(n=129)$

\begin{tabular}{|c|c|c|c|}
\hline & $\begin{array}{l}\text { Migraine } \\
(n=97), n(\%)\end{array}$ & $\begin{array}{l}\text { TTH } \\
(n=32), n(\%)\end{array}$ & $p$ value \\
\hline Relapse & $29(29.9)$ & $3(9.4)$ & 0.020 \\
\hline No relapse & $68(70.1)$ & $29(90.6)$ & \\
\hline
\end{tabular}

$\mathrm{MOH}$, medication overuse headache; TTH, tension-type headache.

Table 2. The relationship between 1-year relapse of $\mathrm{MOH}$ in patients after treatment by education level $(n=129)$

\begin{tabular}{llll}
\hline & $\begin{array}{l}\text { Low level } \\
(n=77), n(\%)\end{array}$ & $\begin{array}{l}\text { High level } \\
(n=52), n(\%)\end{array}$ & $p$ value \\
\hline Relapse & $24(31.2)$ & $8(15.4)$ & 0.042 \\
No relapse & $53(68.8)$ & $44(84.6)$ & \\
\hline
\end{tabular}

Low level, illiteracy, elementary school and middle school; High level, high school, secondary vocational school, 3-year college, a bachelor's degree and above.

$\mathrm{MOH}$, medication overuse headache.

of medication overuse was $5.1 \pm 5.8$ years ( $0.25-40$ years). Eight patients used a single nonsteroidal anti-inflammatory drug (6.2\%), 120 used compound pain relievers (93.0\%), and one used a triptan $(0.8 \%)$.

No significant differences in demographic and clinical characteristics (sex, age, primary headache type, pain reliever type, headache duration, and medication overuse duration) were observed between patients who failed to complete and those who did complete the 12 -month consultation. The reason that 31 patients failed to complete the 12-month consultation was that they did not visit our outpatient clinic.

At the 12-month consultation, 32 patients had relapsed (24.8\%), and 97 patients had not relapsed (75.2\%). The relapse rate was significantly lower in the TTH patients $(3 / 32,9.4 \%)$ than in the migraine patients $(29 / 97$, $29.9 \%$; Table 1). The patients were also divided into 2 categories based on education level: a low education level indicated illiteracy, elementary school, and middle school, whereas a high level indicated high school, secondary vocational school, 3-year college, or a bachelor's degree or higher. The relapse rate was significantly higher in the patients with a low education level $(24 / 77,31.6 \%)$ than in those with a high education level (8/52, 15.4\%; Table 2). 
Table 3. Multifactor unconditional logistic regression analysis results

\begin{tabular}{llllllll}
\hline Name of factor & B & SE & Wald & Df & Significant & Exp(B) & 95\% CI for Exp(B) \\
\hline Primary headache type & 1.313 & 0.652 & 4.059 & 1 & 0.044 & 3.719 & $1.036-13.344$ \\
Education level & 0.803 & 0.465 & 2.985 & 1 & 0.084 & 2.233 & $0.898-5.556$ \\
\hline
\end{tabular}

Table 4. Factors predicting 1 -year relapse of $\mathrm{MOH}$ in patients after successful treatment $(n=129)$

\begin{tabular}{|c|c|c|c|}
\hline & $\begin{array}{l}\text { Relapse } \\
(n=32)\end{array}$ & $\begin{array}{l}\text { Nonrelapse } \\
(n=97)\end{array}$ & $p$ value \\
\hline Gender, $n(\%)$ & & & 0.119 \\
\hline Male & $4(13.8)$ & $25(28)$ & \\
\hline Female & $28(86.2)$ & $72(72)$ & \\
\hline Age, years, mean \pm SD & $49.0 \pm 7.6$ & $46.9 \pm 7.6$ & 0.127 \\
\hline Education level, $n(\%)$ & & & 0.042 \\
\hline Low education level & $24(75.0)$ & $53(54.6)$ & \\
\hline High education level & $8(25.0)$ & $44(45.4)$ & \\
\hline $\mathrm{BMI}, \mathrm{kg} / \mathrm{m}^{2}$, mean $\pm \mathrm{SD}$ & $24.2 \pm 3.0$ & $23.4 \pm 3.8$ & 0.164 \\
\hline Duration of headache, years, mean $\pm S D$ & $18.7 \pm 10.4$ & $15.5 \pm 10.3$ & 0.097 \\
\hline Pain intensity (VAS), mean \pm SD & $7.8 \pm 1.5$ & $7.3 \pm 1.5$ & 0.168 \\
\hline Duration of medication overuse, years, mean \pm SD & $5.2 \pm 4.6$ & $5.1 \pm 6.2$ & 0.366 \\
\hline Medication type, $n(\%)$ & & & 0.632 \\
\hline NSAIDs & $3(9.4)$ & $5(5.2)$ & \\
\hline Opioids & 0 & 0 & \\
\hline Triptan & $1(3.1)$ & 0 & \\
\hline Combination analgesics & $28(87.5)$ & $92(94.8)$ & \\
\hline Primary headache type, $n(\%)$ & & & 0.020 \\
\hline Migraine & 29 & 68 & \\
\hline TTH & 3 & 29 & \\
\hline
\end{tabular}

$\mathrm{MOH}$, medication overuse headache; BMI, body mass index; VAS, visual analog scale; NSAID, nonsteroidal antiinflammatory drug; TTH, tension-type headache.

The primary headache type and education level were included in the logistic regression analysis, and the OR for a primary headache type was $3.719(1.036-13.344, p=$ 0.044; Table 3).

Because only one $\mathrm{MOH}$ patient overused a triptan, we analyzed only those patients who used single and compound pain relievers, among whom we found no significant difference between the relapse and nonrelapse groups. Furthermore, no significant differences were identified among other variables, including body mass index, headache duration and severity, or medication overuse duration (Table 4).

\section{Discussion}

This study assessed the $\mathrm{MOH}$ relapse rate and the risk factors for relapse in the largest sample size in China to date. We found that there were significant differences in the type of primary headache and the education level between the relapse vs. nonrelapse patients and that the type of primary headache was an independent risk factor for $\mathrm{MOH}$ relapse.

In our study, the 1-year relapse rate of $\mathrm{MOH}$ patients was $24.8 \%$, which was close to the relapse rates found by the majority of studies that have been reported globally $[2,8]$. In summarizing 22 studies on the effective treatment rates and relapse rates of $\mathrm{MOH}$ patients, Chiang et al. [9] noted that the effective rates were $60-83 \%$ and the 1 -year relapse rates $17-43 \%$ in the majority of studies with a 1-year follow-up, while a previous study performed by Yan et al. [8] in China reported a 9.5-month relapse rate of $\mathrm{MOH}$ after treatment of $31.4 \%$. In addition, Katsarava et al. [10,11] performed 1- and 4-year follow-ups among $\mathrm{MOH}$ patients after drug withdrawal treatment and found that the relapse rates were 41 and $44 \%$, respectively. The authors proposed that $\mathrm{MOH}$ re- 
lapse mainly occurred in the first year after treatment. Accordingly, we retrospectively assessed $\mathrm{MOH}$ relapse for 1 year.

The type of primary headache is an independent risk factor for $\mathrm{MOH}$ relapse, and patients with migraine as the primary headache are more prone to relapse than are patients with other types. Nonetheless, inconsistent results have been reported with regard for the association between the primary headache type and $\mathrm{MOH}$ relapse. In a study by Katsarava et al. [10], the 1-year relapse rate was significantly lower in patients with migraine as the primary headache type than in patients with TTH as the primary headache (migraine: TTH $=$ 22\%: 73\%), although Sances et al. [12] found that the 1 -year relapse rate of $\mathrm{MOH}$ patients was not associated with the type of primary headache. However, our results showed that the relapse rate was significantly higher in migraine patients than in TTH patients (migraine: $\mathrm{TTH}=29.9 \%: 9.4 \%$ ), contrary to the findings presented in Katsarava et al. [10] The possible reasons for this discrepancy are as follows: the study by Katsarava et al. [10] had a small sample size, with only 11 TTH patients, and there are differences between populations with Eastern and Western ethnicities. Furthermore, 2 previous studies by Diener et al. [2] and Evers et al. [6] both found that migraine was the main primary headache in $\mathrm{MOH}$ patients, indicating that migraine patients are more prone than TTH patients to secondary $\mathrm{MOH}$. Therefore, we hypothesized that the standardized treatment for $\mathrm{MOH}$ patients might lead to a higher relapse rate in migraine patients than in TTH patients, and our study results confirm this hypothesis. Indeed, according to logistic regression analysis, the primary headache type was an independent risk factor for $\mathrm{MOH}$ relapse, and our results indicate that patients with $\mathrm{MOH}$ secondary to TTH might have a lower 1-year relapse rate after treatment than do patients with $\mathrm{MOH}$ secondary to migraine.

A previous study also identified a lower education level as a risk factor for migraine with $\mathrm{MOH}[6,13]$, and in our previous study, the average education level of $\mathrm{MOH}$ patients was lower than that of other $\mathrm{CDH}$ patients (education level below middle school: 64.6 vs. $42.0 \%, p<$ 0.001 ) [14]. The current study shows that patients with a low education level (middle school or below) had significantly higher relapse rates $(24 / 77,31.6 \%)$ than did those with a high education level (high school or beyond; $8 / 52$, 15.4\%). However, logistic regression analysis of education level indicated that it was not an independent risk factor for $\mathrm{MOH}$ relapse.
We found that migraine was the most common primary headache, found in $75.2 \%$ of $\mathrm{MOH}$ patients, which was similar to the $80 \%$ reported in another study in Latin America [7]. Despite the fact that a previous epidemiology study in China reported that the prevalence of TTH was higher than that of migraine (TTH: migraine = 10.8\%: 9.3\%) [3], our results confirm that migraine more commonly induced $\mathrm{MOH}$. Because migraine is usually severe and adversely affects a patient's life, work, and studies, it is highly possible that migraine patients are more prone to frequently overusing acute-pain relievers.

In this study, we found that most $\mathrm{MOH}$ patients used compound pain relievers (93.0\%), consistent with a previous study by Dong et al. [14]. In China, the majority of $\mathrm{MOH}$ patients take compound pain relievers instead of triptans, likely because compound pain relievers are inexpensive, over-the-counter drugs, whereas triptans are prescribed medications that are difficult and more expensive to acquire; for example, triptans can be up to 14 times as expensive as over-the-counter drugs. Jensen et al. [15] found that although single pain relievers and triptans were the pain relievers most commonly used by $\mathrm{MOH}$ patients [7], most $\mathrm{MOH}$ patients in China take compound pain relievers. This discrepancy may be due to the differing conditions in different countries. Most studies thus far have shown that the type of pain reliever is associated with a patient's $\mathrm{MOH}$ prognosis. In a study by Katsarava et al. [10], the relapse rate of $\mathrm{MOH}$ patients taking triptans (17\%) was lower than that of those taking other general pain relievers (52\%), and Sances et al. [12] found that the relapse rate of $\mathrm{MOH}$ patients taking compound pain relievers was significantly higher than that of those using other pain relievers $(33 \%$, $p=0.03)[7,12]$. We were unable to investigate the effects of triptan in this study because only one of our included patients used this type of pain reliever, and our analysis of single versus compound pain relievers did not identify any significant differences, likely because compound pain relievers were taken by the majority of our $\mathrm{MOH}$ patients.

Nonetheless, we did identify a significant sex difference among $\mathrm{MOH}$ patients (female: male $=77.5 \%: 22.5 \%$ ), consistent with an epidemiological study of $\mathrm{MOH}$ patients in Germany performed by Straube et al. [16] (female: male $=74.1 \%: 25.9 \%)$. A previous study by Dong et al. [14] also noted a significant sex difference among $\mathrm{MOH}$ patients in China (female: male $=3.4: 1$ ) [9]. The main reasons for the significant sex difference among $\mathrm{MOH}$ patients are as follows: (1) the most common pri- 
mary headaches that lead to secondary $\mathrm{MOH}$ are migraine and TTH [2]; (2) epidemiological data in China show that more females than males report primary headaches (female: male $=1.8: 1$ ) and that the migraine incidence rate is significantly higher in females than in males (female: male $=2.1: 1$ ) [3]; and (3) migraine accounted for $75.2 \%$ of all primary headaches in the current study and was the main primary headache type for $\mathrm{MOH}$. Because this was a single-center study based on the neurology outpatient clinics of our hospital, we should consider the effects of selection bias and the health care-seeking behavior of patients.

Additionally, a previous study reported that headache severity and frequency and the migraine disease course were all risk factors for $\mathrm{MOH}$ relapse [7], although a study by Katsarava et al. [10] did not find associations between $\mathrm{MOH}$ relapse and the duration of primary headache or the duration of medication overuse. In this study, we analyzed body mass index, the duration of the primary headache, and the severity and duration of medication overuse and found no significant differences.

In our study, among all $\mathrm{MOH}$ patients' consultations $(74 / 234=31 \%)$, one-third failed to adhere to treatment. The main reasons were (1) the patients' excessive concern about the adverse effects of preventive medications (e.g., they refused to or did not take the medication persistently) and (2) the patients' failure to reduce the amount of acute headache medication, so that the treatment effectiveness rates could not be analyzed.

There are some limitations to our study. First, because our hospital is a tertiary care center, the study may include selection bias. Second, because this study is a retrospective study, there could be recall bias, and the results need to be further confirmed in prospective studies.

\section{Conclusions}

The primary headache type is an independent risk factor for $\mathrm{MOH}$ relapse. Patients with migraine as the primary headache are more prone than those with other types to relapse, and females are more prone than males to $\mathrm{MOH}$. Compound pain relievers are the pain relievers most commonly used by $\mathrm{MOH}$ patients in China.

\section{Acknowledgments}

The authors thank all patients who participated in the study.

\section{Statement of Ethics}

This study was approved by the Ethics Committee of Chinese PLA General Hospital.

\section{Disclosure Statement}

There are no potential conflicts of interest with respect to the research, publication of this article, and/or financial completion.

\section{Funding Sources}

This work was supported by the National Natural Science Foundation of China (grants 81771200, 81671077, 81600952, 81771180, 81901134, and 81901145), Beijing Natural Science Foundation Essential Research Project Z170002, for which the authors express gratitude.

\section{Author Contributions}

H.L. contributed to the statistical analysis and writing of the manuscript. Z.D. and S.Y. conducted the research design and modified the manuscript, and others contributed to collect the clinical data.

\section{References}

1 Headache Classification Committee of the International Headache Society (IHS). The International Classification of Headache Disorders, 3rd edition. Cephalalgia. 2018;38(1):1-211.

2 Diener HC, Holle D, Solbach K, Gaul C. Medication-overuse headache: risk factors, pathophysiology and management. Nat Rev Neurol. 2016 Oct;12(10):575-83

3 Yu S, Liu R, Zhao G, Yang X, Qiao X, Feng J, et al. The prevalence and burden of primary headaches in China: a population-based door-to-door survey. Headache. 2012 Apr; 52(4):582-91.
4 Linde M, Gustavsson A, Stovner LJ, Steiner TJ, Barré J, Katsarava Z, et al. The cost of headache disorders in Europe: the Eurolight project. Eur J Neurol. 2012 May;19(5):70311.

5 Vos T, Allen C, Arora M, Barber RM, Bhutta ZA, Brown A, et al.; GBD 2015 Disease and Injury Incidence and Prevalence Collaborators. Global, regional, and national incidence, prevalence, and years lived with disability for 310 diseases and injuries, 1990-2015: a systematic analysis for the Global Burden of Disease Study
2015. Lancet. 2016 Oct;388(10053):1545602 .

6 Evers S, Marziniak M. Clinical features, pathophysiology, and treatment of medication-overuse headache. Lancet Neurol. 2010 Apr;9(4):391-401

7 Shand B, Goicochea MT, Valenzuela R, Fadic R, Jensen R, Tassorelli C, et al.; COMOESTAS CONSORTIUM. Clinical and Demographical Characteristics of Patients with Medication Overuse Headachein Argentina and Chile:Analysis of the Latin American Section of COMOESTAS Project. J Headache Pain. 2015;16(1):83. 
8 Yan Z, Chen Y, Chen C, Li C, Diao X. Analysis of risk factors for medicationoveruse headache relapse: a clinic-based study in China. BMC Neurol. 2015 Sep; 15(1):168.

9 Chiang CC, Schwedt TJ, Wang SJ, Dodick DW. Treatment of medication-overuse headache: A systematic review. Cephalalgia. 2016 Apr;36(4):371-86.

10 Katsarava Z, Limmroth V, Finke M, Diener HC, Fritsche G. Rates and predictors for relapse in medication overuse headache: a 1-year prospective study. Neurology. 2003 May;60(10):1682-3.
11 Katsarava Z, Muessig M, Dzagnidze A, Fritsche G, Diener HC, Limmroth V. Medication overuse headache: rates and predictors for relapse in a 4-year prospective study. Cephalalgia. 2005 Jan;25(1):12-5.

12 Sances G, Ghiotto N, Galli F, Guaschino E, Rezzani C, Guidetti V, et al. Risk factors in medication-overuse headache: a 1-year follow-up study (care II protocol). Cephalalgia. 2010 Mar;30(3):329-36.

13 Atasoy HT, Unal AE, Atasoy N, Emre U, Sumer M. Low income and education levels may cause medication overuse and chronicity in migraine patients. Headache. 2005 Jan; 45(1):25-31.
14 Dong Z, Chen X, Steiner TJ, Hou L, Di H, He $\mathrm{M}$, et al. Medication-overuse headache in China: clinical profile, and an evaluation of the ICHD-3 beta diagnostic criteria. Cephalalgia. 2015 Jul;35(8):644-51.

15 Jensen R, Zeeberg P, Dehlendorff C, Olesen J. Predictors of outcome of the treatment programme in a multidisciplinary headache centre. Cephalalgia. 2010 Oct;30(10):1214-24.

16 Straube A, Pfaffenrath V, Ladwig KH, Meisinger C, Hoffmann W, Fendrich K, et al. Prevalence of chronic migraine and medication overuse headache in Germany-the German DMKG headache study. Cephalalgia. 2010 Feb;30(2):207-13. 